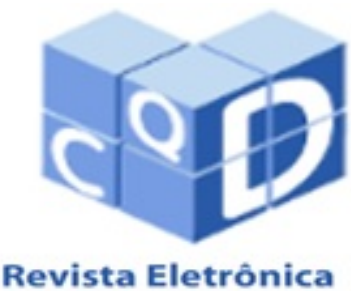

Revista Eletrônica

Paulista de Matemática

ISSN 2316-9664

Volume 16, dez. 2019

Márcio Rostirolla Adames

Universidade Tecnológica

Federal do Paraná - UTFPR

marcioadames@utfpr.edu.br

Marcos Roberto Dalpiaz

Universidade do Vale do Itajaí -

UNIVALE

mrdalpiaz@gmail.com

\section{Uma definição da curva de Koch e prova das suas propriedades fundamentais}

\author{
A definition of the Koch Curve and proof of its fundamental \\ properties.
}

\begin{abstract}
Resumo
Apresentamos uma variação da definição dada por Koch para a curva que leva o seu nome. Com essa definição demonstramos que o conjunto definido é, de fato, uma curva; que a curva não tem tangentes em nenhum ponto; que é formada por cópias reduzidas de si mesma e que apresenta uma estrutura fina. Por fim apresentamos uma definição de dimensão fractal e aplicamos ela à curva de Koch.

Palavras-chave: Fractais. Curva de Koch.
\end{abstract}

\begin{abstract}
We present a variation on the definition given by Koch to the curve that carries its name. With this definition we show that the set defined is, indeed, a curve; that the curve has no tangents; that it is composed of reduced copies of itself and, consequently, also has a fine structure. Lastly we present a definition of fractal dimension and apply it to the Koch Curve.
\end{abstract}

Keywords: Fractals. Koch Curve. 


\section{Introdução}

Os fractais frequentemente despertam o interesse do público devido à beleza e à complexidade das figuras geradas através de iterações de processos simples. Frequentemente utilizamos definições mais intuitivas para definir fractais, pois definições precisas desses objetos podem ser excessivamente complexas para muitos propósitos. Por exemplo a curva de Koch pode ser definida (e.g. a reinterpretação de Adames e Dalpiaz (2016) da definiç ão de Koch) como:

Definição 1. Considere um segmento de reta e fixe um de seus lados. Obtém-se a curva de Koch ao repetir-se infinitas vezes o seguinte processo:

i) dividem-se cada um dos segmentos de reta em três segmentos de igual comprimento;

ii) desenham-se triângulos equiláteros com bases formadas pelos terços médios de cada segmento dividido no primeiro passo, de modo que todos os triângulos estejam do lado da poligonal induzido pela escolha inicial;

iii) apagam-se os segmentos centrais obtidos no primeiro passo.

Tais definições são boas para despertar o interesse e motivar o estudo dos fractais, mas muitas vezes escondem as razões pelas quais o fractal tem suas diversas propriedades e como elas são obtidas. Ressaltamos, nesta perspectiva, que tal definição não deixa claro o que significa "repetir infinitas vezes" o processo descrito. Além disso notamos que uma definição precisa da curva de Koch é incomum em textos sobre fractais e precisamos recorrer ao trabalho original dele, intitulado "Sobre uma Curva Contínua sem Tangentes Construível da Geometria Elementar" " (de Koch (1904) em Edgar(2004)), para encontrar uma definição precisa e que nos permita demonstrar a validade das propriedades.

Nesse trabalho apresentamos, então, uma variação da definição apresentada por Koch. Então demonstramos, inspirados pelas ideias de Koch, que o conjunto definido como a curva de Koch é, de fato, uma curva. Em seguida damos demonstrações de que a curva é auto-similar e que possui uma estrutura fina. Por fim apresentamos uma definição de dimensão fractal e calculamos a dimensão da curva de Koch.

A contribuição desse artigo está em apresentar demonstrações baseadas em conceitos mais simples que os trabalhos clássicos sobre o tema, mas que ainda sejam satisfatórias do ponto de vista da matemática. Não pensamos que esse trabalho deva substituir as formulações mais intuitivas da curva de Koch e suas propriedades, mas servir como referência complementar em língua portuguesa. Um material didático de formulação mais intuitiva é, por exemplo, desenvolvido pelos autores em Adames e Dalpiaz (2016).

\section{Propriedades da curva de Koch}

Dados pontos $P_{0,0}$ e $P_{0,1}$ e o segmento orientado ${ }^{2}, P_{0,0} P_{0,1}$, definido por eles, construiremos uma família de poligonais $P_{k, 0} P_{k, 1} P_{k, 2} \ldots P_{k, j} P_{k, j+1}$, para $k \in\{0,1,2,3, \ldots, n\}$, utilizando a seguinte operação auxiliar:

\footnotetext{
${ }^{1}$ Em tradução livre.

${ }^{2}$ Utilizamos $P_{0,0} P_{0,1}$ ao invés de $\overrightarrow{P_{0,0} P_{0,1}}$ para simplificar a notação, assim como em Koch (1904) em Edgar (2004).
} 
Definição 2. Definimos uma operação $\omega$, que age em segmentos orientados do plano, da seguinte forma: dado um segmento orientado $A B$, definimos $\omega(A B)$ como a poligonal $A C D E B$, definida através do seguinte processo:

1. divide-se o segmento $A B$ em três segmentos de igual comprimento: $A C, C E$ e EB;

2. forma-se um triângulo equilátero $C D E$ sobre o segmento $C E$, de modo que o triângulo esteja do lado esquerdo ${ }^{3}$ induzido pela orientação de $A B$;

3. retira-se o segmento CE.

A Figura 1 ilustra o processo que define a operação $\omega$.

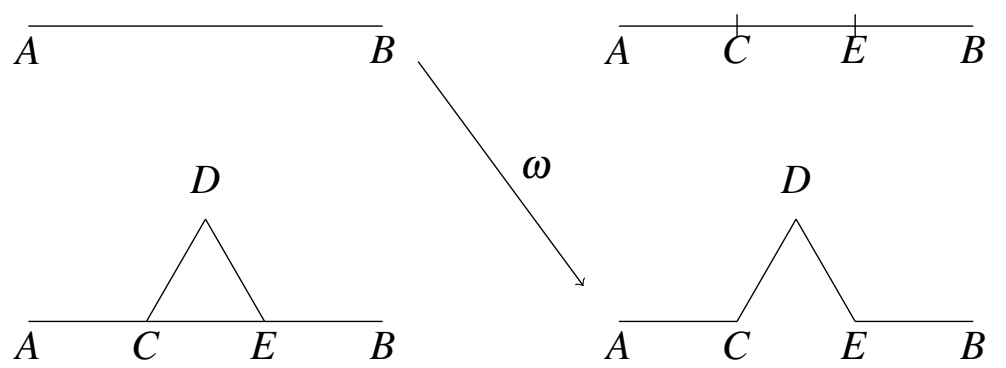

Figura 1 - A operação $\omega$ leva um segmento orientado $A B$ na poligonal $A C D E B$.

Para manter uma notação consistente ao gerarmos a família de poligonais que gera a curva de Koch, escrevemos do seguinte modo: dado o segmento orientado $P_{k, j} P_{k, j+1}$, denotamos a poligonal $\omega\left(P_{k, j} P_{k, j+1}\right)$ por

$$
\omega\left(P_{k, j} P_{k, j+1}\right)=P_{k+1,4 j} P_{k+1,4 j+1} P_{k+1,4 j+2} P_{k+1,4 j+3} P_{k+1,4 j+4}
$$

Note que o ponto final de $\omega\left(P_{k, j-1} P_{k, j}\right)$ e o ponto inicial de $\omega\left(P_{k, j} P_{k, j+1}\right)$ são ambos denotados por $P_{k+1,4 j}$, contudo a notação é consistente pois, da definição de $\omega$, estes pontos não são alterados mas são ambos iguais a $P_{k, j}$.

Definição 3. Sejam $P_{0,0}$ e $P_{0,1}$ pontos e $P_{0,0} P_{0,1}$ o segmento orientado definido por eles. Definimos recursivamente uma família de poligonais $P_{0}, P_{1}, \ldots P_{k}$ por:

i) $P_{0}=P_{0,0} P_{0,1}$;

ii) se $P_{k}=P_{k, 0} P_{k, 1} \ldots P_{k, 4^{k}-1} P_{k, 4^{k}}$ e $\omega\left(P_{k, j} P_{k, j+1}\right)$ é denotado como na Equação (1), então

$$
\begin{aligned}
P_{k+1}= & \omega\left(P_{k, 0} P_{k, 1}\right) \omega\left(P_{k, 1} P_{k, 2}\right) \ldots \omega\left(P_{k, 4^{k}-1} P_{k, 4^{k}}\right) \\
= & P_{k+1,0} P_{k+1,1} P_{k+1,2} P_{k+1,3} P_{k+1,4} \ldots \\
& P_{k+1,4^{k+1}-4} P_{k+1,4^{k+1}-3} P_{k+1,4^{k+1}-2} P_{k+1,4^{k+1}-1} P_{k+1,4^{k+1}}
\end{aligned}
$$

A curva de Koch $P$, gerada a partir do segmento orientado $P_{0,0} P_{0,1}$, pode ser definida do seguinte modo: um ponto $x \in \mathbb{R}^{2}$ está na curva de Koch se, e somente se, $x$ é o limite de uma sequência 4 $\left(x_{k}\right)$, com $x_{k} \in P_{k}$ para todo $k \in \mathbb{N}$. 


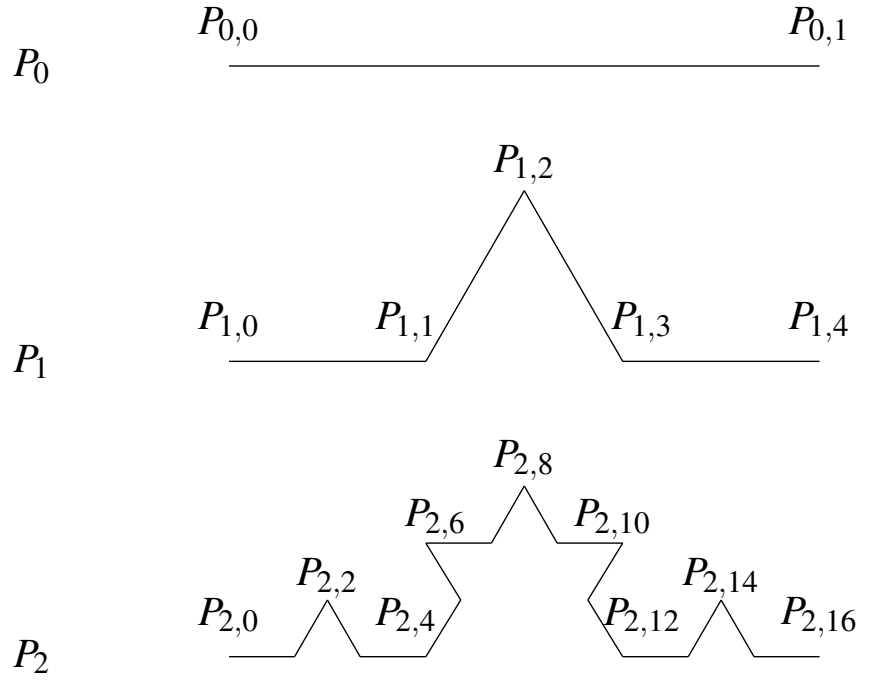

Figura 2 - As primeiras poligonais obtidas ao aplicarmos repetidamente a operação $\omega$.

A Figura 2 ilustramos a família de poligonais utilizada para definir a curva de Koch.

Observação 4. Ressaltamos que a definição é consistente. Isso se deve ao fato de a operação $\omega$ não alterar o terço inicial e o terço final dos segmentos no qual é aplicada, de modo que, se $P_{k, j-1} P_{k, j}$ e $P_{k, j} P_{k, j+1}$ são segmentos consecutivos de $P_{k}$, então $\omega\left(P_{k, j-1} P_{k, j}\right)$ e $\omega\left(P_{k, j} P_{k, j+1}\right)$ são poligonais com o mesmo ponto final e inicial, respectivamente. Portanto aplicando $\omega$ a cada segmento de uma poligonal e unindo todas as poligonais obtidas obtemos, de fato, uma outra poligonal (e não várias poligonais disjuntas).

Observação 5. Também pelo fato de $\omega$ não alterar o terço inicial e o terço final dos segmentos no qual é aplicada, o ângulo entre os segmentos final de $\omega\left(P_{k, j-1} P_{k, j}\right)$ e inicial de $\omega\left(P_{k, j} P_{k, j+1}\right)$ é o mesmo que o ângulo entre os segmentos $P_{k, j-1} P_{k, j}$ e $P_{k, j} P_{k, j+1}$ e os pontos $P_{k, j-1}, P_{k, j}$ e $P_{k, j+1}$ não são colineares. Assim $P_{k}$ é uma poligonal formada por $4^{k}$ segmentos e com $4^{k}+1$ vértices.

Lema 6. Todos os segmentos de $P_{k}$ têm comprimento ${ }^{5} P_{0,0} P_{0,1} / 3^{k}$.

Demonstração. A demonstração segue por indução. Primeiro notemos que $P_{0}=P_{0,0} P_{0,1}$, que tem comprimento $P_{0,0} P_{0,1} / 3^{0}$. Assumimos, como hipótese de indução, que o segmento $P_{k, j} P_{k, j+1}$ tem comprimento $P_{0,0} P_{0,1} / 3^{k}$ para todo $j \in\left\{0,1,2, \ldots, 4^{k}-1\right\}$. Pela definição da poligonal $P_{k+1}$, qualquer um de seus segmentos é um segmento de $\omega\left(P_{k, j} P_{k, j+1}\right)$ para algum $j \in\left\{0,1,2, \ldots, 4^{k}-\right.$ $1\}$ e, deste modo, terá comprimento igual a $1 / 3$ do comprimento de $P_{k, j} P_{k, j+1}=P_{0,0} P_{0,1} / 3^{k}$ e, portanto, tem comprimento igual a $P_{0,0} P_{0,1} / 3^{k+1}$.

Corolário 7. O comprimento de $P_{k}$ é $P_{0,0} P_{0,1}\left(\frac{4}{3}\right)^{k}$.

\footnotetext{
${ }^{3}$ Para fixar a orientação.

${ }^{4}$ Koch (1904) em Edgar (2004), por outro lado, considera o conjunto de vértices de todas as poligonais, simultaneamente, e define a curva como o conjunto dos pontos de acumulação desse conjunto. Preferimos a definição através do limite da sequência, pois ela capta a sequência de poligonais presente na ideia intuitiva do conjunto. Não é difícil ver que as definições são equivalentes.

${ }^{5}$ Usamos $P_{0,0} P_{0,1}$ para denotar um segmento e também para denotar o seu comprimento, assim como Koch, acreditando que o contexto deixa claro sobre qual dos dois estamos nos referindo.
} 
Demonstração. Segue do número de segmentos na Observação 5 e do comprimento de cada um destes segmentos no Lema 6.

Observação 8. Uma das preocupações de Koch foi mostrar que o conjunto chamado de curva de Koch na Definição 3 é, de fato, uma curva contínua. Para isso ele constrói uma função contínua do intervalo inicial na curva. Para definirmos tal função fazemos uso de duas famílias de funções, definidas abaixo.

i) Sejam $P_{k, j} P_{k, j+1} o(j+1)$-ésimo segmento da k-ésima poligonal na Definição 3 e $\vec{\eta}_{k, j} o$ vetor normal a este segmento que aponta para a esquerda do segmento, de acordo com a direção induzida pela orientação do segmento inicial $P_{0,0} P_{0,1}$.

ii) Seja $x \in P_{0,0} P_{0,1}$. Definimos uma sequência $\left(x_{k}\right)$, com $x_{k} \in P_{k}$, recursivamente através de:

$$
\begin{aligned}
& x_{0}=x ; \\
& x_{k+1}=x_{k}+f_{k}(x) \vec{\eta}_{k, j} ;
\end{aligned}
$$

onde $x_{k} \in P_{k, j} P_{k, j+1}$ e $f_{k}(x) \in[0, \infty)$ é o único número real não negativo tal que $x_{k}+f_{k}(x) \vec{\eta}_{k, j} \in$ $\omega\left(P_{k, j} P_{k, j+1}\right) \subset P_{k+1}$.

iii) Deixando x variar na sequência do item anterior obtém-se famílias de funções $f_{k}: P_{0,0} P_{0,1} \rightarrow$ $[0, \infty) e \vec{v}_{k}: P_{0,0} P_{0,1} \rightarrow \mathbb{R}^{2}$ definida por

$$
\vec{v}_{k}(x)=x_{0}+\sum_{n=1}^{k} f_{k}(x) \vec{\eta}_{k, j} .
$$

De modo que $\vec{v}_{k}(x)=x_{k}$ para qualquer $x \in P_{0,0} P_{0,1}$. Note que $f_{k} e \vec{v}_{k}$ são contínuas, para qualquer $k \in \mathbb{N}$, pela definição de $\omega$ e pelo fato de que $f_{k}(x)=0$ sempre que $x_{k}$ está nos terços inicial e final de qualquer segmento da poligonal $P_{k}$.

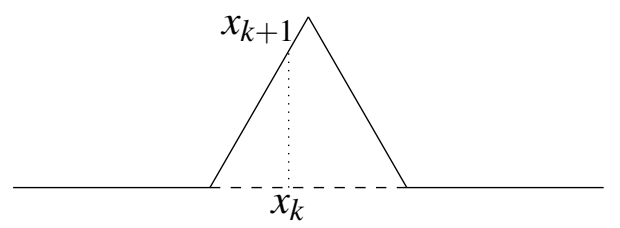

$$
\begin{gathered}
P_{k, j} P_{k, j+1} \cdots \\
\omega\left(P_{k, j} P_{k, j+1}\right)-
\end{gathered}
$$

Figura 3 - Distância entre um ponto $x_{k} \in P_{k, j} P_{k, j+1}$ em um segmento da $k$-ésima poligonal e sua imagem na próxima iteração da poligonal $x_{k+1} \in \omega\left(P_{k, j} P_{k, j+1}\right)$.

Lema 9. As funções $\sum f_{k} e \vec{v}_{k}$ convergem uniformemente em $P_{0,0} P_{0,1}$. E, em particular, a sequência $x_{k}=\vec{v}_{k}(x)$ é convergente para qualquer $x \in P_{0,0} P_{0,1}$.

Demonstração. Sejam $x \in P_{0,0} P_{0,1}, k \in \mathbb{N}$ e $x_{k}=\vec{v}_{k}(x)$. Além disso seja $P_{k, j} P_{k, j+1}$ o segmento que contém o ponto $x_{k}$. Note que, pelo Lema $6, P_{k, j} P_{k, j+1}=P_{0,0} P_{0,1} / 3^{k}$. O ponto $x_{k+1}$ é definido através de $\omega\left(P_{k, j} P_{k, j+1}\right)$ e a distância $d\left(x_{k}, x_{k+1}\right)$ é menor do que a altura do triângulo formado por $\omega$ a partir de $P_{k, j} P_{k, j+1}$, como ilustrado na Figura 3 .

Deste modo

$$
f_{k}(x)=d\left(x_{k}, x_{k+1}\right) \leq \frac{P_{0,0} P_{0,1}}{6.3^{k}} \operatorname{tg}\left(\frac{\pi}{3}\right)=\frac{P_{0,0} P_{0,1}}{6.3^{k}} \sqrt{3} .
$$


Assim

$$
\left\|\vec{v}_{k}(x)\right\| \leq \sum_{n=1}^{k}\left|f_{n}(x)\right| \leq P_{0,0} P_{0,1} \sum_{n=0}^{k}\left(\frac{1}{6.3^{n}} \sqrt{3}\right) \leq \frac{\sqrt{3}}{6} P_{0,0} P_{0,1} \sum_{n=0}^{\infty} \frac{1}{3^{n}} .
$$

Como a série numérica da direita converge, o teste $M$ de Weierstrass garante a convergência uniforme de $\sum f_{k} \mathrm{e} \vec{v}_{k}$.

Observação 10. Sejam então $f=\sum f_{k} e \vec{v}=\lim _{k \rightarrow \infty} \vec{v}_{k}$. Ambas são contínuas pois são limites de funções contínuas que convergem uniformemente. Além disso $\vec{v}\left(P_{0,0} P_{0,1}\right) \subset P$, onde $P$ é a curva de Koch, pois $\vec{v}_{k}(x) \in P_{k}$ para todo $k \in \mathbb{N}$.

Vamos mostrar que qualquer ponto de $P$ está na imagem da função $\vec{v}$.

Lema 11. Dado um ponto qualquer y de P e uma sequência $\left(y_{k}\right)$ com $y_{k} \in P_{k}$ para todo $k \in \mathbb{N}$, tal que $y_{k} \longrightarrow y \in P$, existe $x \in P_{00} P_{01}$ tal que $\vec{v}(x)=y$.

Demonstração. Para $y_{k} \in \omega\left(P_{k-1, j} P_{k-1, j+1}\right) \subset P_{k}$ existe um único ponto, que denotamos proj ${ }_{P_{k-1}}^{\omega} y_{k}$, em $P_{k-1, j} P_{k-1, j+1}$ tal que $y_{k}$ está na perpendicular a $P_{k-1, j} P_{k-1, j+1}$ que passa por $\operatorname{proj}_{P_{k-1}}^{\omega} y_{k}$. Tomando $k$ projeções consecutivas obtemos o ponto $z_{k}=\operatorname{proj}_{P_{0}}^{\omega} y_{k} \in P_{0,0} P_{0,1}$. Assim $z_{k} \in P_{0,0} P_{0,1}$ é tal que a sequência $\left(\vec{v}_{n}\left(z_{k}\right)\right)_{n \in \mathbb{N}}$ tem $y_{k}$ como $k$-ésimo termo, como ilustra a Figura 4.

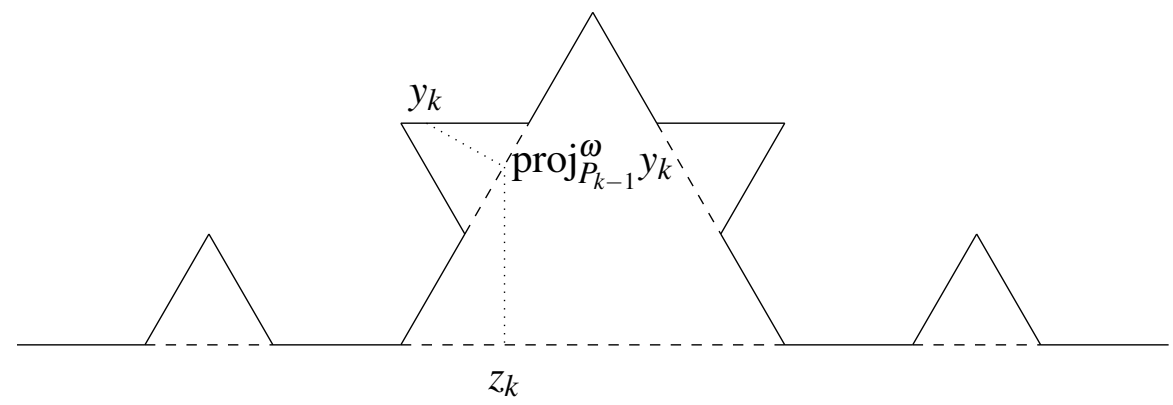

Figura 4 - Sequência em $P_{0,0} P_{0,1}$ gerada a partir de $y_{k}$.

A sequência $\left(z_{k}\right)$ está contida no compacto $P_{0,0} P_{0,1}$ e, portanto, admite uma subsequência convergente $\left(z_{k_{i}}\right)$. Seja $x=\lim _{i \rightarrow \infty} z_{k_{i}}$.

Por outro lado, a imagem $\vec{v}\left(P_{0,0} P_{0,1}\right)$ do compacto $P_{0,0} P_{0,1}$ também é compacta, pois $\vec{v}$ é contínua, e a sequência $\vec{v}\left(z_{k_{i}}\right)$ admite uma subsequência convergente que denotaremos da mesma forma: $\vec{v}\left(z_{k_{i}}\right)$. Seja $y^{*}=\lim _{i \rightarrow \infty} \vec{v}\left(z_{k_{i}}\right)$.

Agora observe que para qualquer $x \in P_{0,0} P_{0,1}$ e para quaisquer $m, p \in \mathbb{N}$ temos

$$
\begin{aligned}
& \left\|\vec{v}_{m}(x)-\vec{v}_{m+p}(x)\right\| \leq \sum_{n=m}^{m+p}\left|f_{n}\right| \leq P_{0,0} P_{0,1} \sum_{n=m}^{m+p} \frac{1}{6.3^{n}} \operatorname{tg}\left(\frac{\pi}{3}\right) \\
& \left\|\vec{v}_{m}(x)-\vec{v}_{m+p}(x)\right\| \leq \frac{P_{0,0} P_{0,1}}{6.3^{m}} \operatorname{tg}\left(\frac{\pi}{3}\right) \sum_{n=0}^{\infty} \frac{1}{3^{n}}=\frac{P_{0,0} P_{0,1}}{4.3^{m}} \sqrt{3}
\end{aligned}
$$

$\mathrm{e}$

$$
\left\|\vec{v}_{m}(x)-\vec{v}(x)\right\| \leq \frac{P_{0,0} P_{0,1}}{4.3^{m}} \sqrt{3}
$$

Deste modo, dado $\varepsilon>0$, existe $n_{0} \in \mathbb{N}$ tal que $i>n_{0}$ implica em: 
i) $d\left(y, y_{k_{i}}\right)<\varepsilon / 3$, pois $y_{k} \rightarrow y$;

ii) $d\left(y_{k_{i}}, \vec{v}\left(z_{k_{i}}\right)\right)<\varepsilon / 3$, pela Eq. (2), que vale para todo $x$, e devido ao fato que $y_{k_{i}}=\vec{v}_{k_{i}}\left(z_{k_{i}}\right)$;

iii) $d\left(\vec{v}\left(z_{k_{i}}\right), y^{*}\right)<\varepsilon / 3$, pois $\vec{v}\left(z_{k_{i}}\right) \rightarrow y^{*}$;

portanto, para qualquer $\varepsilon>0$, temos

$$
d\left(y, y^{*}\right) \leq d\left(y, y_{k_{i}}\right)+d\left(y_{k_{i}}, \vec{v}\left(z_{k_{i}}\right)\right)+d\left(\vec{v}\left(z_{k_{i}}\right), y^{*}\right)<\varepsilon
$$

e $y=y^{*}$

Observação 12. Assim, a curva de Koch é, de fato, uma curva, no sentido de que é a imagem de um intervalo através de uma função contínua. Contudo, a Observação 7 mostra que o comprimento das poligonais $P_{k}$ tende ao infinito quando $k \rightarrow \infty$, apesar da curva estar contida em uma região finita do plano. Deste modo, é natural dizer que o comprimento da curva de Koch $P$ é infinito.

Vejamos que a curva de Koch não contém tangentes. Para isto mostramos que em qualquer ponto de $P$ não há limite para as retas secantes de $P$, no sentido que em qualquer vizinhança de um ponto $x \in P$ existem pontos $y \in P$ e $z \in P$ tais que a secante definida por $x$ e $y$ e a secante definida por $x$ e $z$ encontram-se em ângulo $\theta$, ângulo este que não está nem muito próximo de zero, nem muito próximo de $\pi$.

Lema 13. Sejam $x \in P$ e $x_{0} \in P_{0,0} P_{0,1}$ tais que $x_{k}:=\vec{v}_{k}\left(x_{0}\right) \rightarrow x$ quando $k \rightarrow \infty$. Existem constantes $\varepsilon_{1}$ e $\varepsilon_{2}$ tais que, dado $\varepsilon>0$, existem $y_{k} \in P$ e $z_{k} \in P \operatorname{com} d\left(x, y_{k}\right)<\varepsilon$ e d $\left(x, z_{k}\right)<\varepsilon$ com as secantes $x y_{k}$ e $x z_{k}$ encontrando-se em ângulo $\theta$ tal que $0<\varepsilon_{1}<\theta<\varepsilon_{2}<\pi$.

Demonstração. Suponha que $x_{k} \in P_{k, j} P_{k, j+1}$. Pelo Lema 6, temos que $P_{k, j} P_{k, j+1}=P_{0,0} P_{0,1} / 3^{k}$. Por outro lado, a Equação (2)

$$
d\left(x, P_{k, j} P_{k, j+1}\right) \leq d\left(x, x_{k}\right) \leq \frac{P_{0,0} P_{0,1}}{4.3^{k}} \sqrt{3}=: d_{k} .
$$

Desta forma $x$ está contido na $d_{k}$-vizinhança de $P_{k, j} P_{k, j+1}$, que dividimos nas regiões $\alpha, \beta$ e $\gamma$, como ilustram as Figuras 5 e 6.

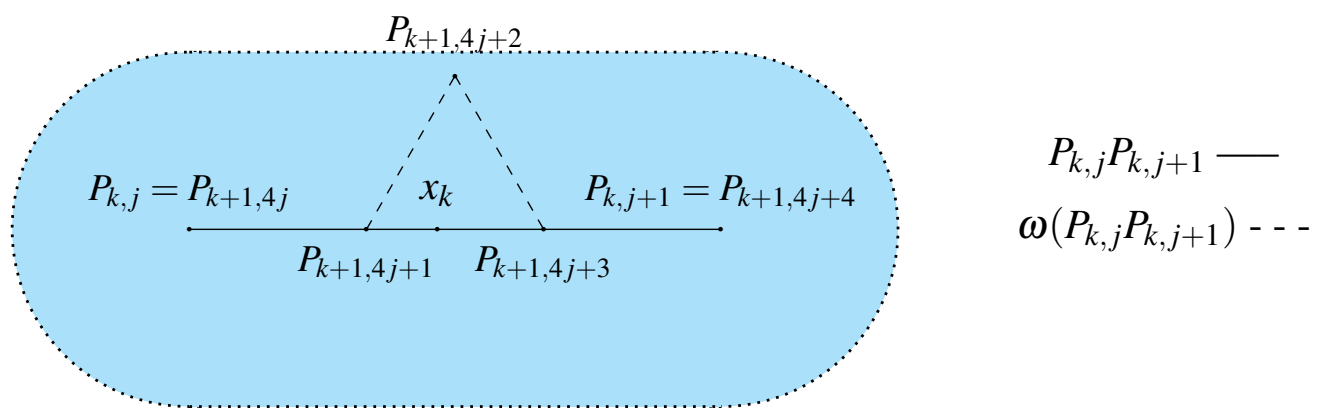

Figura 5 - A figura ilustra a $d_{k}$-vizinhança de um segmento da $k$-ésima poligonal $P_{k, j} P_{k, j+1}$, que contém todos os pontos obtidos da curva de Koch gerada a partir de $P_{k, j} P_{k, j+1}$. 


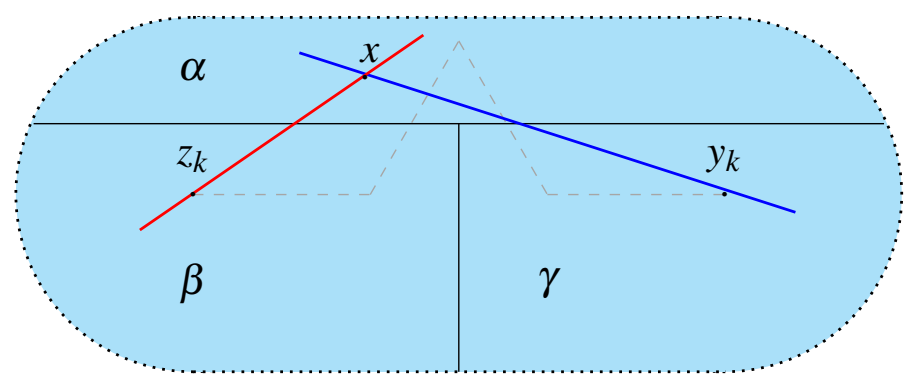

Figura 6 - A $d_{k}$-vizinhança dividida em três regiões caracterizando retas concorrentes que não se aproximam de paralelas.

Se o ponto $x \in P$ está na região $\alpha$, tomamos $y_{k}=P_{k, j}=P_{k+1,4 j}$ e $z_{k}=P_{k, j+1}=P_{k+1,4 j+4}$, de modo que o ângulo $\theta$ entre as retas $x y_{k}$ e $x z_{k}$ satisfaz as condições requeridas, como ilustra a Figura 6.

Se o ponto $x \in P$ está na região $\beta$, tomamos $y_{k}=P_{k+1,4 j+2}$ e $z_{k}=P_{k, j+1}=P_{k+1,4 j+4}$, de modo que o ângulo $\theta$ entre as retas $x y_{k}$ e $x z_{k}$ satisfaz as condições requeridas.

Se o ponto $x \in P$ está na região $\gamma$, tomamos $y_{k}=P_{k, j}=P_{k+1,4 j}$ e $z_{k}=P_{k+1,4 j+2}$, de modo que o ângulo $\theta$ entre as retas $x y_{k}$ e $x z_{k}$ satisfaz as condições requeridas.

Note, contudo, que para todo $k \in \mathbb{N}$ os pontos $y_{k}$ e $z_{k}$ são vértices de $\omega\left(P_{k, j} P_{k, j+1}\right)$ e, portanto, $y_{k} \rightarrow x$ e $z_{k} \rightarrow x$. Além disso eles são vértices da poligonal $P_{k+1}$ e, portanto, pontos de $P$, devido ao fato de $\omega$ não alterar os vértices dos segmentos no quais é aplicada. Deste modo, temos pares de pontos de $P$, tão próximos quanto desejarmos de $x$, tais que o ângulo $\theta$ entre as secantes não está muito próximo de zero ou de $\pi$.

Vamos mostrar agora que a operação curva de Koch é formada por 4 cópias de si mesma. Para isso é necessário observarmos que a operação $\omega$ não é alterada por movimentos rígidos.

Definição 14. Um movimento rígido é qualquer transformação afim gerada pela composição finita de rotações, translações e reflexões.

Observação 15. Sejam $A B$ e $C D$ segmentos de comprimento a e $b$ respectivamente. Seja $\mathscr{F}$ : $\mathbb{R}^{2} \rightarrow \mathbb{R}^{2}$ a composição de um movimento rígido com uma homotetia em algum ponto do plano, de modo que $\mathscr{F}$ é uma bijeção do segmento $A B$ sobre o segmento $C D$, com A sobre $C$ e $B$ sobre $D$. Se $\omega(A B)$ e $\omega(C D)$ são as poligonais obtidas por uma iteração do processo que gera a curva de Koch, então a função

$$
\left.\mathscr{F}\right|_{\omega(A B)}: \omega(A B) \rightarrow \omega(C D),
$$

é uma bijeção. Isso segue do fato que movimentos rígidos e homotetias preservam ângulos e retas, levando triângulos semelhantes em triângulos semelhantes. A Figura 7 ilustra a aplicação.

Proposição 16. Sejam $A B$ e $C D$ segmentos orientado de comprimento a e $b$, respectivamente. Seja $\mathscr{T}: \mathbb{R}^{2} \rightarrow \mathbb{R}^{2}$ o movimento rígido que leva o segmento $A B$ sobre o segmento $C D$, com $A$ sobre $C$. Além disso, seja $\mathscr{H}: \mathbb{R}^{2} \rightarrow \mathbb{R}^{2}$ a homotetia de razão $\frac{b}{a}$ e centro em $C$. Se $P(A B) e$ $P(C D)$ são as curvas de Koch produzidas a partir dos segmentos $A B$ e $C D$, respectivamente, então a função $\mathscr{F}:=\mathscr{H} \circ \mathscr{T}: P(A B) \rightarrow P(C D)$, é uma bijeção.

Demonstração. Vamos iniciar mostrando por indução que $\mathscr{F}$ é uma bijeção entre as poligonais $P_{k}(A B)$ e $P_{k}(C D)$ obtidas por $k$ aplicações de $\omega$ sobre os segmentos $A B$ e $C D$. 
i) A transformação afim $\mathscr{T}$ leva o segmento $A B$ no segmento $A^{\prime} B^{\prime}$, de forma que $A^{\prime}=C$ e $B^{\prime}$ está na semirreta $\overrightarrow{C D}$. Como movimentos rígidos preservam distâncias, o comprimento de $A^{\prime} B^{\prime}$ é $a$. Então a homotetia $\mathscr{H}$ leva o segmento $A^{\prime} B^{\prime}$ no segmento $A^{\prime \prime} B^{\prime \prime}$ de forma que $A^{\prime \prime}=A^{\prime}=C$ e o comprimento de $A^{\prime \prime} B^{\prime \prime}$ é

$$
a \frac{b}{a}=b
$$

Assim, $C D$ e $A^{\prime \prime} B^{\prime \prime}$ são segmentos de mesma origem, sobre a mesma semirreta e de mesmo comprimento. Portanto, $C D$ e $A^{\prime \prime} B^{\prime \prime}$ são idênticos e têm a mesma orientação.

ii) Assumimos, como hipótese de indução, que a função $\mathscr{F}$ é uma bijeção entre as poligonais $P_{k}(A B)$ e $P_{k}(C D)$, onde $P_{k}(A B)$ e $P_{k}(C D)$ são as $k$-ésimas poligonais obtidas a partir dos segmentos $A B$ e $C D$ respectivamente.

Em particular, cada um dos $4^{k}$ segmentos da $k$-ésima iteração sobre $A B$ é levado, através de uma bijeção, sobre um dos $4^{k}$ segmentos da $k$-ésima iteração de $C D$. Contudo, aplicar $\omega$ à poligonal $P_{k}(A B)$ é o mesmo que aplicar uma iteração em cada um dos $4^{k}$ segmentos de $P_{k}$. A Observação 15 garante que a mesma função $\mathscr{F}$ é uma bijeção entre as poligonais obtidas por uma iteração nos respectivos segmentos.

Mostramos agora que $\mathscr{F}$ também é uma bijeção entre as curvas de Koch $P(A B)$ e $P(C D)$.

Em primeiro lugar, $\mathscr{F}$ associa cada ponto de $P(A B)$ a um ponto de $P(C D)$. De fato: um ponto $x$ está em $P(A B)$ se, e somente se, existir uma sequência $\left(x_{k}\right) \operatorname{com} x_{k} \in P_{k}(A B)$ e $\lim x_{k}=x$. Como $\mathscr{F}$ é uma bijeção entre $P_{k}(A B)$ e $P_{k}(C D)$ para qualquer $k \in \mathbb{N}$, a sequência $\left(x_{k}\right)$ define uma sequência de pontos $\left(\mathscr{F}\left(x_{k}\right)\right)$, onde $\mathscr{F}\left(x_{k}\right)$ está em $P_{k}(C D)$. Todavia, $\mathscr{F}$ é a composição de um movimento rígido com uma homotetia e, em particular, é contínua. Assim, $\mathscr{F}\left(x_{k}\right)$ converge para $y \in P(C D)$, pela definição da curva de Koch. Analogamente, qualquer ponto de $P(C D)$ é levado por $\mathscr{F}^{-1}$ em um ponto de $P(A B)$.

Em segundo lugar, $\mathscr{F}$ associa pontos distintos de $P(A B)$ a pontos distintos de $P(C D)$. Como $\mathscr{F}$ é a composição de um movimento rígido (que preserva a distância) com uma homotetia de razão $\frac{b}{a}$, temos que para $x, x^{\prime} \in \mathbb{R}^{2}$

$$
d\left(x, x^{\prime}\right)>0 \Rightarrow d\left(\mathscr{F}(x), \mathscr{F}\left(x^{\prime}\right)\right)=\frac{b}{a} d\left(x, x^{\prime}\right)>0
$$

Deste modo $x \neq x^{\prime} \Rightarrow \mathscr{F}(x) \neq \mathscr{F}\left(x^{\prime}\right)$.

A Proposição 16 mostra que curvas de Koch produzidas por segmentos distintos diferem apenas pelo tamanho ${ }^{6}$ e por um movimento rígido.

\footnotetext{
${ }^{6}$ Devido à homotetia.
}

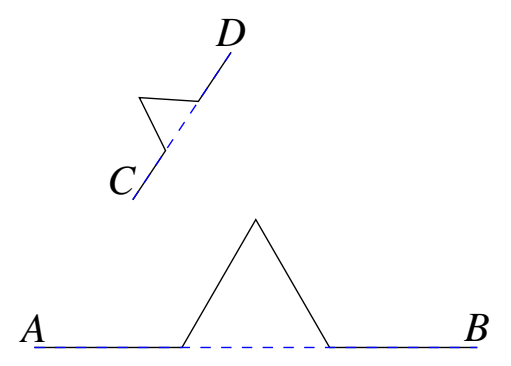

Figura 7 - A primeira iteração da operação $\omega$ em dois segmentos distintos $A B$ e $C D$. 
Proposição 17. A curva de Koch é formada por 4 cópias de si mesma, com cada uma das cópias reduzidas por uma homotetia de razão um terço no plano.

Demonstração. Ao aplicarmos a primeira iteração ao segmento inicial obtemos 4 segmentos, cada um deles com 1/3 do tamanho do segmento original. Aplicar uma iteração do processo na Definição 3 é o mesmo que aplicá-lo às 4 poligonais geradas a partir dos 4 segmentos, pois o processo age de maneira independente sobre cada segmento. Pela Proposição 16, o processo gerará 4 curvas de Koch idênticas, sendo que cada uma delas pode ser obtida da curva original por uma homotetia de razão 1/3 composta com um movimento rígido.

Observação 18. A curva de Koch é dita auto-similar devido à Proposição 17, que mostra que a curva é formada por cópias de si mesma. Além disso, podemos entendê-la da seguinte forma: a curva de Koch é formada por 4 cópias de si mesma, cada uma delas com um terço do tamanho original; cada uma destas cópias é formada por 4 cópias de si mesma, agora com um nono do tamanho original; e assim sucessivamente. Deste modo, partes tão pequenas quanto desejarmos são formadas por cópias reduzidas da curva original e, portanto, ainda retém toda a complexidade desta. Ademais, o Lema 13 mostra que a curva não contém tangentes em nenhum ponto $e$, portanto, nenhuma de suas partes é semelhante aquelas da geometria euclidiana (que contém tangentes); o que pode ser entendido como uma estrutura fina presente na curva.

Discutimos agora a dimensão da curva de Koch. Uma das maneiras pela qual a dimensão de um objeto geométrico manifesta-se matematicamente é através do fato que objetos de dimensão diferente comportam-se de modo diferente quando homotetias são aplicadas. Por exemplo: uma homotetia de razão $k \in \mathbb{N}$ leva um segmento em outro segmento em outro segmento, que é formado $k$ cópias do segmento original; uma homotetia de razão $k$ leva um retângulo em outro retângulo, que é formado por $k^{2}$ cópias do retângulo original; e uma homotetia de razão $k$ leva um cubo em outro cubo, que é formado por $k^{3}$ cópias do cubo original, como ilustra a Figura 8.

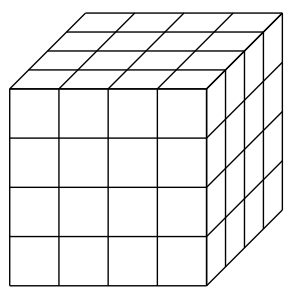

Figura 8 - Uma homotetia de razão 4 em um cubo gera $4^{3}=64$ cópias dele.

Assim a dimensão está no expoente da razão de homotetia no número de cópias obtidas. Isso nos motiva a definir a dimensão de um objeto geométrico que seja auto-similar como:

Definição 19. Se realizando uma homotetia de razão $k$ no plano obtém-se $n$ cópias do fractal X, definimos a dimensão fractal deste fractal como

$$
\operatorname{dim} X=\log _{k} n .
$$

Exemplo 20. Pela Proposição 17, a curva de Koch contém 4 cópias de si mesma, cada uma delas com 1/3 do tamanho da curva original. Isso significa que, se realizarmos uma homotetia de razão 3 no plano, obtemos 4 cópias da curva de Koch. Assim a curva tem dimensão

$$
\operatorname{dim} X=\log _{3} 4 \approx 1,261859507 .
$$


Por fim ressaltamos que não definimos no texto o que é um fractal. Uma definição é: um conjunto $X$ é um fractal se sua dimensão topológica é menor do que a dimensão de Hausdorff Besicovitch, como definido em Mandelbrot (1982). Contudo esta definição exclui alguns conjuntos que gostaríamos de chamar de fractais, segundo Falconer (1990), que pensa em fractais sem uma definição formal, mas em conjuntos que tem, tipicamente, algumas características como:

1. $X$ tem uma estrutura fina, isto é, detalhes em escalas arbitrariamente pequenas;

2. $X$ é muito irregular para ser descrito em linguagem geométrica tradicional;

3. $X$ tem alguma forma de auto-similaridade;

4. a "dimensão fractal" não é, na maioria dos casos, inteira;

5. $X$ está definido de maneira simples na maioria dos casos de interesse, talvez recursivamente.

Fractais em geral não precisam ter auto-similaridade exata, isto é, que pequenas partes sejam cópias reduzidas exatas do todo, de modo que a dimensão apresentada não é suficiente para todos os casos. O estudo de fractais em geral e suas dimensões tem teorias mais elaboradas e o presente material é apenas um ponto de partida para este estudo.

\section{Conclusão}

No texto apresentamos algumas das propriedades fundamentais da curva de Koch, que muitas vezes não estão presentes em textos de divulgação sobre fractais. Não pretendemos indicar aqui que toda a exposição sobre o assunto deva ser formal, de fato nós mesmos produzimos um material com uma discussão mais informal sobre o assunto em Adames e Dalpiaz (2016). Nosso objetivo com o presente artigo é de colaborar com a divulgação das razões que justificam as propriedades dos fractais; razões estas que tivemos dificuldades para encontrar em textos em português. Ambos os trabalhos estão relacionados à dissertação Dalpiaz (2016) do PROFMAT.

\section{Agradecimentos}

O presente trabalho foi realizado com apoio da Coordenação de Aperfeiçoamento de Pessoal de Nível Superior - Brasil (CAPES) - Código de Financiamento 001.

\section{Referências bibliográficas}

ADAMES, M. R; DALPIAZ, M. R. Investigando fractais no ensino básico: uma proposta paradidática. Curitiba: UTFPR, 2016. 40p. 2016. Disponível em:

http://www.utfpr.edu.br/curitiba/estrutura-universitaria/diretorias/dirppg/programas/profmat/ produtos-e-projetos-do-profmat-utct. Acesso em: 18 de jul. de 2019.

DALPIAZ, M. R. Um estudo sobre fractais: origem e proposta didática para aplicação em aula. 72f. 2016. Dissertação (Mestrado) - Universidade Tecnológica Federal do Paraná. Programa de Mestrado Profissional em Matemática em Rede Nacional, Curitiba, 2016. 
$\mathrm{KOCH}, \mathrm{H}$. On a continuous curve without tangents constructible from elementary geometry. In: EDGAR, G. A. (ed.). Classics on fractals. Boulder: Westview Press, 2004. p. 25-45.

FALCONER, K. Fractal geometry: mathematical foundations and applications. Chichester: John Wiley \& Sons, 1990.

MANDELBROT, B. B. The fractal geometry of nature. New York: W. H. Freeman \& Company, 1982. 